At NFXL1, an Ar abi dopsi s homol ogue of the human transcription fact or NF-X1, functions as a negat i ve regul at or of the tri chothecene phyt ot oxi n- i nduced def ense response

\begin{tabular}{|c|c|}
\hline 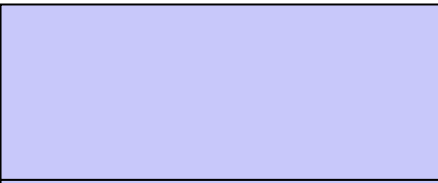 & $\begin{array}{l}\text { Asano Tomøa, Nasuda Dai suke, Yasuda M chi ko, } \\
\text { Nakashi ta H deo, Kudo Toshi aki, Ki mur a Nakoto, } \\
\text { Yamaguchi Kazuo, Ni shi uchi Takumi }\end{array}$ \\
\hline 著者別表示 & 浅野 智哉, 山口 和男, 西内 巧 \\
\hline $\begin{array}{l}\text { j our nal or } \\
\text { publ i cat i on titl e }\end{array}$ & $\mathrm{Pl}$ ant Journal \\
\hline vol une & 53 \\
\hline nunber & 3 \\
\hline page $r$ ange & 450-464 \\
\hline year & $2008-02-01$ \\
\hline URL & ht t p: //doi . or g/10. 24517/00028405 \\
\hline
\end{tabular}




\section{the plant journal}

AtNFXL1, an Arabidopsis homologue of the human transcription factor NF-X1, functions as a negative regulator of the trichothecene phytotoxininduced defense response.

\begin{tabular}{|r|l|}
\hline Journal: & The Plant Journal \\
\hline Manuscript ID: & TPJ-00751-2007.R1 \\
\hline Manuscript Type: & Full Paper \\
\hline Date Submitted by the & n/a \\
\hline Complete List of Authors: & $\begin{array}{l}\text { Asano, Tomoya; Kanazawa University, Advanced Science Research } \\
\text { Center } \\
\text { Masuda, Daisuke; Kanazawa University, ASRC } \\
\text { Yasuda, Michiko; RI KEN, Environmental Molecular Biology } \\
\text { Laboratory } \\
\text { Nakashita, Hideo; RI KEN, Environmental Molecular Biology } \\
\text { Laboratory } \\
\text { Kudo, Toshiaki; RI KEN, Environmental Molecular Biology Laboratory } \\
\text { Kimura, Makoto; RI KEN, Discovery Research Institute (DRI), Plant } \\
\text { \& Microbial Metabolic Engineering Research Unit } \\
\text { Yamaguchi, Kazuo; Kanazawa University, ASRC } \\
\text { Nishiuchi, Takumi; Kanazawa university, ASRC }\end{array}$ \\
\hline Key Words: & $\begin{array}{l}\text { trichothecene, phytotoxin, microarray, transcription factor, } \\
\text { Fusarium, defense response, SA biosynthesis }\end{array}$ \\
\hline & \\
\hline
\end{tabular}

\section{s) ScholarONE


AtNFXL1, an Arabidopsis homologue of the human transcription factor NF-X1, functions as a negative regulator of the trichothecene phytotoxin-induced defense response.

Tomoya Asano ${ }^{\mathbf{1}} \mathbf{2}$, Daisuke Masuda ${ }^{\mathbf{1}}$, Michiko Yasuda ${ }^{\mathbf{3}}$, Hideo Nakashita ${ }^{\mathbf{3}}$, Toshiaki Kudo $^{3}$, Makoto Kimura ${ }^{4}$, Kazuo Yamaguchi ${ }^{1,5}$ and $*$ Takumi Nishiuchi ${ }^{1,5}$

${ }^{1}$ Division of Functional Genomics, Advanced Science Research Center, Kanazawa University, 13-1 Takaramachi, Kanazawa 920-0934, Japan; ${ }^{2}$ Shigeta Animal Pharmaceuticals Inc., 4569-1, Komoridani, Oyabe City, Toyama Prefecture, 932-0133 Japan; ${ }^{3}$ Environmental Molecular Biology Laboratory, RIKEN, 2-1 Hirosawa, Wako, Saitama 351-0198, Japan; ${ }^{4}$ Plant \& Microbial Metabolic Engineering Research Unit, Discovery Research Institute (DRI), RIKEN, 2-1 Hirosawa, Wako, Saitama 351-0198, Japan; ${ }^{5}$ Division of Life Science, Graduate School of Natural Science and Technology, Kanazawa University, Kanazawa 920-1192, Japan

*Author for correspondence (e-mail: tnish9@kenroku.kanazawa-u.ac.jp)

total word count: $\mathbf{7 , 1 3 4}$ 
Running title: Trichothecene-inducible AtNFXL1 gene

key words: phytotoxin, trichothecene, transcription factor, defense response 


\begin{abstract}
Trichothecenes are a closely related family of phytotoxins produced by phytopathogenic fungi. In Arabidopsis, expression of AtNFXL1, a homologue of the putative human transcription repressor $N F-X 1$, was significantly induced by application of type A trichothecenes, such as T-2 toxin. An atnfxll mutant growing on medium lacking trichothecenes showed no phenotype, whereas a hypersensitivity phenotype was
\end{abstract} observed in T-2 toxin-treated atnfxll mutant plants. Microarray analysis indicated that several defense-related genes (i.e. WRKYs, NBS-LRRs, EDS5, ICS1, etc.) were upregulated in T-2 toxin-treated atnfxll mutant compared to wild type plants. In addition, enhanced salicylic acid (SA) accumulation was observed in T-2 toxin-treated atnfxl1 mutant plants, which suggests that AtNFXL1 functions as a negative regulator of these defense-related genes via an SA-dependent signaling pathway. We also found that expression of AtNFXL1 was induced by SA and flg22 treatment. Moreover, the atnfxll mutant was less susceptible to a compatible phytopathogen, Pseudomonas syringae pv. tomato strain DC3000 (Pst DC3000). Taken together, these results indicate 
that AtNFXL1 plays an important role in the trichothecene response, as well as the general defense response in Arabidopsis. 


\section{Introduction}

Trichothecenes are a major type of mycotoxin, and are important in human health due to the risk of ingesting contaminated food (Kimura et al., 2006). Phytopathogenic fungi capable of producing trichothecenes are found throughout the world, and include certain species of Fusarium, Myrotherium and Stachybotrys (Eudes et al., 2001). The production of mycotoxins by these species of phytopathogenic fungi is determined by genetic factors and environmental growth conditions. Trichothecenes have a sesquiterpenoid ring structure, and can be classified according to the presence or absence of characteristic functional groups (Shifrin and Anderson, 1999). Type A trichothecenes, such as T-2 toxin, and type B trichothecenes, such as deoxynivalenol (DON), are natural contaminants of certain agricultural commodities, as well as commercial foods (Sudakin, 2003). Among the trichothecenes, type A trichothecenes are highly toxic at low concentrations.

Trichothecenes inhibit peptidyltransferase activity in eukaryotic cells by binding to the $60 \mathrm{~S}$ ribosomal subunit. The antiproliferative activity of trichothecenes is presumed to be a consequence of their ability to inhibit protein synthesis (Shifrin and 
Anderson, 1999). Thus, trichothecenes also function as phytotoxins. Specific disruption of a trichothecene synthase gene (Tri5) in F. graminearum resulted in a strain that was less virulent in the infection of wheat compared to wild type strains (Desjardins et al., 2000). For this reason, Desjardins et al. have suggested that in certain Fusarium species, trichothecenes act as virulence factors in the infection of plants (Desjardins et al., 2000). Trichothecene-producing Fusarium species have strain-specific trichothecene metabolite profiles (Ward et al., 2002), and these trichothecene chemotypes are also believed to play a role in the virulence of individual strains of Fusarium.

Recently, we reported that type A trichothecenes, such as T-2 toxin, have an elicitor-like activity in Arabidopsis thaliana at a concentration of $1 \mu \mathrm{M}$ (Nishiuchi et al., 2006). Type A trichothecene-inducible lesions were also formed in SA-, jasmonic acid (JA)- and ethylene (ET)-mutants, and in SA-deficient NahG transgenic plants (Nishiuchi et al., 2006). These results implied that T-2 toxin-induced cell death has little to do with these host defense pathways; rather, the toxin contributes directly to the virulence of necrotrophic phytopathogens. In contrast to T-2 toxin, $10 \mu \mathrm{M}$ DON inhibited protein translation in Arabidopsis cells, whereas it failed to activate the 
elicitor-like signaling pathway (Nishiuchi et al., 2006), which suggests that Fusarium utilizes DON as a non-defense-inducing translational inhibitor during the spread of disease in host plants (Bai et al., 2001). Thus, the role of type B trichothecenes in virulence might be different from that of type A trichothecenes. Urban et al. reported that the DON-producing, wheat-attacking fungal pathogens $F$. graminearum and $F$. culmorum can infect the flowers of Arabidopsis contaminated with DON (Urban et al., 2002).

We recently reported that AtNFXL1 is upregulated in T-2 toxin-treated Arabidopsis (Masuda et al., 2007). AtNFXL1 encodes a putative transcription factor with similarity to the human transcription repressor NF-X1 (Lisso et al., 2006). Human NF-X1 was identified as a binding factor for the conserved $\mathrm{X} 1$ box regulatory element in the proximal promoters of class II $M H C$ genes, and contains a nuclear localization signal (NLS), a RING-CH finger domain, several NF-X1-type zinc (Zn) finger domains, and an R3H domain (Song et al., 1994). Song et al. suggested that NF-X1 is involved in regulating disease states by suppressing the expression of class II $M H C$ genes (Song et al., 1994). The RING-CH finger domain is implicated in the targeting of proteins for 
ubiquitination (Lorick et al., 1999). The yeast $N F-X 1$ homologue, FAP1, was identified in a genetic screen for suppressors of rapamycin toxicity (Kunz et al., 2000). FAP1 interacted physically with a FK506-binding protein 12 (FKBP12) in vivo and in vitro, and suppressed the cytotoxic effects of rapamycin (Kunz et al., 2000). Strombakis et al. suggested that the Drosophila NF-X1 homologue, shuttle craft (stc), is essential for embryogenesis by regulating the activity of a subset of genes that play a role in either the guidance or spatial maintenance of axon tracts (Strombakis et al., 1996). Taken together, these results suggest that the NF-X1 family of proteins has unique functions in different organisms.

In this paper, we demonstrated that atnfxll mutant plants exhibit a hypersensitivity phenotype to a type A trichothecene, T-2 toxin. Microarray analysis revealed that many defense-related genes are upregulated in the atnfxll mutant in the presence of trichothecenes, compared to wild type plants. High levels of SA accumulated in T-2 toxin-treated atnfxll mutant plants compared to wild type plants, which suggests that AtNFXL1 functions as a negative regulator of defense-related genes via an SA-dependent signaling pathway. In addition, we found that the expression of AtNFXL1 
is induced by application of SA. Moreover, the atnfxll mutant was less susceptible to the compatible phytopathogen Pst DC3000. Thus, AtNFXL1 also appears to play an important role in the defense response to compatible phytopathgens in Arabidopsis.

\section{Results}

\section{AtNFXL1 belongs to the NF-X1 family of proteins}

Based on its predicted amino acid sequence, AtNFXL1 encoded a protein with a molecular weight of $130 \mathrm{kDa}$ that has similarity to the human transcription repressor NF-X1 (Supplemental Figures 1a and b). AtNFXL1 contains several functional regions and domains, including an NLS, a RING-CH finger domain, and nine NF-X1-type Zn finger domains (Supplemental Figure 1a). These domains are also conserved in Oryza sativa OsNF-X1, Homo sapiens NF-X1, Drosophila melanogaster STC, and Saccharomyces cerevisiae FAP1. The R3H domain, which is involved in binding of single stranded RNA, is present only in NF-X1 family proteins of non-plant eukaryotes (Supplemental Figure 1a). Phylogenetic analysis indicated that plant NF-X1-like proteins are more closely related to human NF-X1 than to FAP1 or STC (Supplemental 
Figure 1b). AtNFXL1 contains an intron in its 5'UTR (data not shown). The NF-X1-type $\mathrm{Zn}$ finger domains are unique motifs, and the $\mathrm{Zn}$ finger repeats are conserved in AtNFXL1 (Supplemental Figure 1c) . It has been reported that a green fluorescent protein (GFP)-AtNFXL1 fusion protein localizes to the nucleus in onion epidermal cells (Lisso et al., 2006). We also examined the localization of a GFP-AtNFXL1 fusion protein in Arabidopsis, and found that GFP-AtNFXL1 localizes to the nucleus in Arabidopsis T87 suspension cultured cells (Supplemental Figure 2).

\section{The atnfxl1 mutant displays a hypersensitivity phenotype to the type A}

\section{trichothecene, T-2 toxin.}

We recently demonstrated that AtNFXL1 is a trichothecene-inducible gene (Masuda et al., 2007). To determine the function of AtNFXL1, we investigated the trichothecene response of atnfxl1 (atnfxl1-1) mutant plants. The atnfxl1-1 mutant was generated by transferred-DNA (T-DNA) insertion at position $+2,082$ (relative to the first basepair of the initiation codon at +1 ) of the open reading frame of AtNFXL1 (Munich Information Center for Protein Sequence designation At1g10170), as previously described (Figure 
1a; Lisso et al., 2007). In wild type plants, AtNFXL1 was weakly expressed in the absence of T-2 toxin, whereas it was induced by $1 \mu \mathrm{M}$ T-2 toxin treatment, as previously reported (Figure 1b; Masuda et al., 2007). In the atnfxll mutant, we observed a truncated transcript of AtNFXL1 (Figure 1b). The deduced amino acid sequence of the truncated mRNA in the atnfxll mutant lacked two of the nine NF-X1-type Zn finger domains. Therefore, it is likely that the truncated form of atnfxll mRNA in mutant plants does not encode a functional protein. The atnfxll mutant exhibited no apparent phenotype on MS agar medium alone (without trichothecene) compared to wild type plants (Figures 1c and 1d). In addition, general phenotypes, such as growth rate, organ development, and morphology of untreated atnfxll mutant were similar to wild type plants (data not shown). In contrast, atnfxll mutant exhibited a severe growth defect on MS medium containing $0.1 \mu \mathrm{M}$ T-2 toxin (Figures 1c and 1d). As previously reported (Masuda et al., 2007), cell death was not induced when seedlings were transferred to 0.1-1 $\mu \mathrm{M} \quad \mathrm{T}-2$ toxin-containing medium. The $\mathrm{T} 2$ segregation ratio of the toxin-hypersensitivity phenotype was nearly $1: 3$ in self-pollinated offspring of heterozygous atnfxll plants, which indicated that the mutation was inherited as a single 
recessive trait. As shown in Figure 1d, the growth defects of DON-treated atnfxl1 mutant were similar to DON-treated wild type plants.

To determine whether the T-2 toxin-sensitive phenotype of atnfxll mutant plants was due to a defect in AtNFXL1, we carried out a complementation analysis. Introduction of a complementation plasmid containing the promoter and the coding sequence of AtNFXL1 (AtNFXL1 promoter::AtNFXL1, see Experimental Procedures) into atnfxl1 mutant plants clearly rescued the hypersensitivity phenotype in the presence of $0.1 \mu \mathrm{M}$ T-2 toxin in 7 of 8 plant lines (Figures $1 \mathrm{c}$ and $1 \mathrm{~d}$ ). These results demonstrated that the hypersensitivity to T-2 toxin of atnfxll mutant plants was due to a defect in AtNFXL1.

Defense-related genes are upregulated in trichothecene-treated atnfxl1 mutant plants.

We performed a transcriptome analysis of approximately 14,880 genes to obtain the expression profiles of putative AtNFXL1-regulated genes. This analysis was carried out using two independent wild-type plants, and two independent mutant plant lines. As 
seen in Figure 1b, atnfxll mutant plants displayed no visible phenotype in the absence of trichothecenes. In accordance with this result, none of the genes we examined were upregulated more than 3-fold in atnfxll mutant plants compared to wild type plants in the absence of trichothecenes (data not shown). A single gene was down-regulated greater than 3-fold in atnfxll mutant plants compared to wild type plants (data not shown). These results indicated that in the absence of trichothecenes, AtNFXL1 has a minor effect on the global regulation of gene expression.

In contrast, in $1 \mu \mathrm{M}$ T-2 toxin-treated atnfxl1 mutant plants, 130 genes were upregulated greater than 3-fold compared to T-2 toxin-treated wild type plants (Table 1). As seen in Table 1, 18 of the upregulated genes were putative transcriptional regulators. In particular, $8 W R K Y$ family genes were upregulated in T-2 toxin-treated atnfxll mutant plants. WRKY transcription factors play pivotal roles in the plant defense response (Eulgem et al., 2000), and expression of some WRKY family genes confers enhanced disease resistance in Arabidopsis and tobacco (Asai et al., 2002; Liu et al., 2004; Chen and Chen, 2002). .

The largest category of putative AtNFXL1-regulated genes (28 genes) encoded 
cellular communication and signal transduction factors (Table 1). This category included 9 genes that encode serine/threonine protein kinases, including a Pto-like kinase, and 7 genes that encode receptor-like protein kinases, which suggests that these genes function as components of AtNFXL1-regulated defense signaling pathways. Several defense-related genes also appeared to be regulated by AtNFXL1, including 5 genes that encode disease resistance proteins, as well as EDS5 and ICS1. EDS5 was identified as an essential component of SA-dependent signaling in resistance to Pst DC3000 in Arabidopsis (Nawrath et al., 2002). ICS1 encodes an isochorismate synthase, and is required for biosynthesis of SA (Wildermuth et al., 2001). These results suggested that AtNFXL1 is involved in SA-dependent defense signaling pathways in trichothecene-treated Arabidopsis.

Table 2 lists the genes that were down-regulated greater than 3-fold in T-2 toxin-treated atnfxll mutant plants compared to wild type plants. The list of genes included $L H C B 2-4$, which suggests that hyperactivation of the defense response affects the expression of phytosynthesis-related genes.

To validate the results of the microarray analysis, we selected 6 genes that 
were upregulated, and 1 gene that was down-regulated in T-2 toxin-treated atnfxll mutant plants, and analyzed them by real time PCR. As shown in Table 3, we obtained similar results using real time PCR, although the magnitude of the expression change of some of the genes was greater than what was observed by microarry analysis.

\section{Enhanced SA accumulation in T-2 toxin-treated atnfxl1 mutant plants.}

Microarray analysis revealed that defense-related genes, including genes involved in SA biosyntheis, were upregulated in atnfxll mutant compared to wild type plants. $P R-1$ (At2g14160), which is regulated in an SA-dependent manner, was not present on the Agilent Arabidopsis 1 microarray. When we examined the expression of $P R-1$ by RT-PCR, we found that $P R-1$ was weakly induced 24 hours (hr) after T-2 toxin treatment in both wild type and atnfxll mutant, as previously described (Masuda et al., 2007). The T-2 toxin-induced expression of ICS1 was enhanced in atnfxl1 mutant plants compared to wild type plants (Figure 2a). These results suggested that SA biosynthesis is activated in atnfxll mutant plants. We next measured free and total SA levels in wild type and atnfxll mutant plants in the presence or absence of $\mathrm{T}-2$ toxin. As seen in 
Figures $2 \mathrm{~b}$ and $2 \mathrm{c}, \mathrm{T}-2$ toxin-induced SA accumulation was enhanced in atnfxll mutant plants compared to wild type plants. Taken together, these results suggested that enhanced SA accumulation in atnfxll mutant plants leads to the induction of defense-related genes (Table 1).

\section{SA and flg22 activate the transcription of AtNFXL1.}

To investigate the expression pattern of AtNFXL1 in more detail, we generated transgenic plants carrying an AtNFXL1 promoter:: $\beta$-glucuronidase (GUS) gene fusion construct. As shown in Figure 3a, in seedlings of AtNFXL1::GUS transformants, in the absence of trichothecene, GUS activity was present in the vascular bundle and meristematic tissue. AtNFXL1 promoter activity was increased up to approximately 18-fold by $0.1 \mu \mathrm{M}$ T-2 toxin treatment compared to mock (no trichothecene) treatment (Figures 3a, 3b and 3d). Treatment with $2.5 \mu \mathrm{M}$ DAS induced an 8-fold increase in promoter activity, while treatment with $10 \mu \mathrm{M}$ DON resulted in a 3-fold induction of promoter activity (Figure 3d). Since AtNFXL1 is predicted to play a role in defense signaling, including SA-dependent signaling, we also investigated whether other 
elicitors and defense-related signals affected the expression of AtNFXL1. AtNFXL1 promoter activity was increased approximately 5-fold by flg22, a peptide elicitor derived from phytopathogenic bacteria (Figure 3d). SA treatment induced an approximate 40-fold increase in GUS activity in AtNFXL1 promoter::GUS transformants (Figure 3a, 3c, and 3d), and 1-aminocyclopropane-1-carboxylic acid (ACC) and methyl jasmonate (MeJA) induced a 2.5-fold and 3.2-fold increase in promoter activity, respectively (Figure 3d). These results suggested that AtNFXL1 plays a role not only in the action of trichothecenes, but also in the general defense response of Arabidopsis.

\section{The atnfxl1 mutant is less susceptible to Pst DC3000.}

To determine whether AtNFXL1 is involved in disease resistance to phytopathogens, wild type and atnfxll mutant plants were inoculated with the compatible pathogen Pst DC3000. As shown in Figure 4a, the growth of Pst DC3000 in atnfxll mutant plants was slower than in wild type plants, which indicated that atnfxl1 mutant plants are less susceptible to Pst DC3000. The reduced susceptibility to the compatible pathogen Pst 
DC3000 was not observed after complementation with wild type AtNFXL1 (Figure 4b).

These results indicated that the reduced susceptibility phenotype of atnfxll mutant is due to a defect in AtNFXL1. These results also provided further evidence that AtNFXL1 functions not only in the trichothecene response, but also in the general defense response in Arabidopsis.

\section{Discussion}

The action of trichothecenes in host plants can not simply be attributed to general toxicity, such as inhibition of translation. For example, we previously reported that some type A trichothecenes have an elicitor-like activity in infiltrated Arabidopsis leaves (Nishiuchi et al., 2006). Both DON and DAS preferentially inhibit root elongation, whereas T-2 toxin-treated seedlings exhibit dwarfism and aberrant morphological changes (Masuda et al., 2007). In contrast, neither feature was observed in seedlings treated with a general translational inhibitor, cycloheximide (CHX). These results indicate that the action of trichothecenes in plants differs significantly according to molecular species, and highlight the importance of examining the site of 
action of trichothecenes in host plants. In this study, we demonstrated that AtNFXL1 is an important regulator of trichothecene action in Arabidopsis. Our results may provide a key to understanding the molecular mechanism of phytotoxic trichothecenes in host plants.

AtNFXL1 was upregulated not only by type A trichothecenes, but also SA and flagellin (Figure 3). SA, in particular, drastically induced the expression of AtNFXL1. We identified several putative AtNFXL1-regulated genes using microarray analysis, including many defense-related genes, such as WRKYs, RLKs, and NBS-LRRs (Table 1). Since these genes are putative regulators of defense signaling pathways in Arabidopsis, it is likely that AtNFXL1 functions as a component of these pathways, particularly the SA-dependent signaling pathway. Dong et al. reported that many of the Arabidopsis WRKY family genes are induced by pathogen-infection and/or SA treatment, including the putative AtNFXL1-regulated WRKY genes that we identified in the current study (Dong et al., 2003). Overexpression of WRKY6 and WRKY53 results in a dwarfed phenotype in transgenic plants (Robatzek and Somssich, 2002; Ulker and Somssich, 2004); thus, upregulation of these two genes in atnfxll mutant plants may contribute to 
the severe growth defects of these plants in the presence of type A trichothecenes. EDS5, which is an essential component of SA-dependent signaling in resistance to Pst DC3000 in Arabidopsis (Nawrath et al., 2002), and ICS1, which encodes an isochorismate synthase that is required for biosynthesis of SA (Wildermuth et al., 2001), were also upregulated in T-2 toxin-treated atnfxl1 mutant plants. In fact, AtNFXL1 appeared to be involved in the negative regulation of SA biosynthesis in response to T-2 toxin (Figures $2 \mathrm{~b}$ and $2 \mathrm{c}$ ), and possibly other elicitors and infectious pathogens as well. In this manner, AtNFXL1 may act to suppress the hyperactivation of defense responses to elicitors or pathogens. In support of this hypothesis, atnfxll mutant plants displayed less susceptibility to the compatible phytopathogen Pst DC3000 (Figure 4). The atnfxll mutant could not repress the defense response induced by type A trichothecenes, resulting in severe growth defects in trichothecene-treated Arabidopsis seedlings. This phenotype was similar to that of the constitutive defense response mutant cprl (Bowling et al., 1994).

Lisso et al. reported that AtNFXL1 is induced by salt stress and osmotic stress, and that atnfxll mutant plants display reduced survival rates after salt stress compared 
to wild type plants (Lisso et al., 2006). In addition, certain salt-responsive genes, such as COR15A, KIN1, and RAB18, showed weaker expression levels in atnfxll mutant under salt stress compared to the wild type plants (Lisso et al., 2006). The expression of COR15A, KIN1, and RAB18 is also induced by ABA in Arabidopsis (Baker et al., 1994; Kurkela and Franck, 1990; Lang and Palva, 1992). In contrast, transgenic 35S::AtNFXL1 plants exhibited an enhanced survival rate under salt stress, and higher expression of salt-responsive genes. These results indicate that AtNFXL1 functions as a positive regulator of expression of salt-inducible genes under salt stress conditions (Figure 5). We demonstrated that AtNFXL1 negatively regulates the expression of several defense-related genes in trichothecene-treated Arabidopsis plants (Figure 5). Thus, it seems likely that AtNFXL1 has opposing functions in the salt stress response and defense response. ABA plays a negative role in defense signaling pathways, including SA-, JA-, and ET-dependent signaling pathways (Mauch-Mani and Mauch, 2005). Therefore, AtNFXL1-controlled stress signaling might depend on components of both the defense and the ABA signaling pathways.

Human NF-X1 binds directly to cis-elements in target genes in vitro, and 
regulates transcription through these elements in vivo (Song et al., 1994; Gewin et al., 2004). However, activation or repression domains have not been identified in any NF-X1 family protein to date. AtNFXL1 contains a RING-CH finger domain, which is a binding motif for the ubiquitin-conjugating enzyme E2s (Lorick et al., 1999). Thus, AtNFXL1 may function as a repressor by mediating the degradation of its binding partners. NF-X1 exists as two isoforms: NFX1-123 and NFX1-91. Recently it was shown that NFX1-123 and c-Myc function cooperatively to activate the hTERT promoter, whereas NFX1-91 repressed hTERT promoter activity (Gewin et al., 2004). These results raise the possibility that NF-X1 family proteins function as negative regulators of their targets. In support of this hypothesis, Lisso et al. reported that another Arabidopsis NF-X1-like protein, AtNFXL2, is a negative regulator of the salt stress response (Lisso et al. 2006). It has been reported that some elicitor-responsive RING-H2 finger proteins have roles in plant defense signaling pathways (Takai et al., 2002; Serrano and Guzman, 2004). Thus, the RING-CH finger domain of AtNFXL1 may have a role in regulating the stability of defense-related target proteins.

NF-X1 represses INF- $\gamma$-inducible expression of class II $M H C$ genes in 
INF- $\gamma$-treated cells, whereas it has no effect on the expression of these genes in untreated cells (Song et al. 1994). In addition, FAPl was identified as a suppressor of rapamycin toxicity. FAP1 physically interacts with FKBP12 in vivo and in vitro to suppress the function of rapamycin, and FAP1 is targeted to the nucleus by rapamycin treatment. In the current study, we showed that atnfxll mutant plants are hypersensitive to the type A trichothecene, T-2 toxin (Figure 2), but display no phenotype in the absence of chemical. Taken together, these results suggest that AtNFXL1, NF-X1, and FAP1 are together involved responding to chemical stimuli, but have no apparent phenotype in the absence of chemicals.

In summary, we have presented evidence that the trichothecene-inducible gene AtNFXL1 negatively regulates many defense-related genes, at least in part through the regulation of SA biosynthesis (Figure 5). Additional studies that investigate how atnfxl1 mutant behave when challenged by necrotrophic pathogens, such as trichothecene-producing fungi, are needed. While we have not established a Fusarium-Arabidopsis pathosystem for interaction studies, it has been reported that $A$. thaliana is susceptible to type B DON-producing species of Fusarium (Uraban et al., 
2002). Studies to determine whether Arabidopsis is susceptible to T-2 toxin-producing fungi such as Fusarium spoichiomerdes are ongoing, and will further our understanding of the role of AtNFXL1 in host plant resistance to trichothecene-producing fungi.

\section{Experimental procedures}

\section{Plant growth and trichothecene treatment}

The Columbia (Col-0) ecotype of Arabidopsis thaliana (L.) Heynh was used as the wild type plant in this study. Sterile seeds were sown on Murashige and Skoog (MS) medium that contained 3\% (w/v) sucrose and $0.3 \%(w / v)$ gelrite (San-Ei Gen F.F.I., Inc.) in plastic petri dishes, and then stratified for 2 days (d) at $4^{\circ} \mathrm{C}$ in the dark. Plants were grown at $22^{\circ} \mathrm{C}$ under long day conditions (16 hours (hr) light/8 hr dark cycles or continuous light) in a growth chamber. A T-DNA insertion mutant (atnfxl1-1) of AtNFXL1 (N501399) was obtained from the Arabidopsis Biological Resource Center, Ohio State University, Columbus, Ohio. For trichothecene or defense-related molecule treatment, Arabidopsis seeds were sown on MS agar medium containing the indicated 
substance, and plants were continuously grown. Alternatively, Arabidopsis plants were first grown on MS medium without treatment, and then transferred to MS medium containing the indicated molecules. Additional details of each treatment are noted in the text or figure legends.

\section{Generation of transgenic plants}

A region of the AtNFXL1 promoter (-795 basepairs relative to the start site at +1 ) was amplified by PCR using primers

(5'-GCGGGATCCATTCTGCCTTGACTCCACAAA-3'), and then introduced into the HindIII and BamHI sites of pBI121. For complementation analysis, a SacI fragment of the F14N23 BAC clone containing the promoter region and coding region of AtNFXL1 was introduced into the SacI site of pSMAH621. Plasmids were introduced into wild type or atnfxll mutant plants by in planta transformation, as previously described (Asano et al., 2004). Several independent transformants were obtained, and detailed analysis was carried out on T2 and T3 plants. 


\section{Reverse transcription-polymerase chain reaction (RT-PCR) analysis}

In a total volume of $20 \mu \mathrm{l}$, cDNAs were synthesized from $1 \mu \mathrm{g}$ of total RNA using SuperScript III reverse transcriptase (Invitrogen) with a oligo(dT) $)_{16}$ primer, and then 0.5 $\mu l$ of the cDNA was subsequently used for PCR analysis. All PCR reactions were performed in a total volume of $10 \mu \mathrm{l}$, for $24-28$ cycles under the following conditions: denaturation, $94^{\circ} \mathrm{C}, 30$ seconds (s); annealing, $55^{\circ} \mathrm{C}, 30 \mathrm{~s}$; extension, $72^{\circ} \mathrm{C}, 30 \mathrm{~s}$. The following gene-specific primers were used: AtNFXL1 120-438, 5'CCCATATGCCTCCTAATACAGATAGAAATTC-3' 5'-ACGTCGACCTCAGGAGCATTATTTCTTCTATG-3'; AtNFXL1 2363-3568, 5'CGCCATATGCATGTGGTCGTATAACCGCTA-3’ and 5'-GACGTCGACCTCACATACCTTCTCCCAGT-3'; ACT2/8, 5'CATCACACTTTCTACAATGAGCT-3' and 5'-CGACCTTAATCTTCATGCTGC-3'.

\section{Real time PCR analysis}

Real time PCR was performed using the LightCycler Quick System 350S (Roche 
Diagnostics K.K., Tokyo, Japan) with SYBR Premix Ex Taq (TAKARA BIO INC., Shiga, Japan). The PCR reaction contained 1 x SYBR Premix Ex Taq, $0.2 \mu \mathrm{M}$ of each primer, and the appropriate dilution of cDNA in a final volume of $20 \mu \mathrm{l}$. The following PCR program was used: initial denaturation, $95^{\circ} \mathrm{C}, 10 \mathrm{~s} ; 40$ cycles of $95^{\circ} \mathrm{C}, 5 \mathrm{~s}$ and $60^{\circ} \mathrm{C}$, $20 \mathrm{~s}$ with a temperature transition rate of $20^{\circ} \mathrm{C} / \mathrm{s}$; melting curve analysis, $95^{\circ} \mathrm{C}, 0 \mathrm{~s}, 65^{\circ} \mathrm{C}$, $15 \mathrm{~s}$, and an increase to $95^{\circ} \mathrm{C}$ with a temperature transition rate of $0.1^{\circ} \mathrm{C} / \mathrm{s}$. To generate a standard curve, homologous standards were used as external standards in all experiments. Template DNA was quantified using the second derivative maximum methods of the LightCycler Software Ver.3.5 (Roche Diagnostics), then normalized to Actin2/8 mRNA. The following gene-specific primers were used: At5g25930, 5'ACATTGCTCCAGAATACGC-3' and 5'-CATCGCCTCAGTCGTG-3'; WRKY15, 5'-TGCTCGAAGAAAAGAAAGATAAAAC-3' ${ }^{\prime}$ and 5' AGTAACAATCAACATGGACG-3'; At5g41750, 5'-AAAGGAACAGGTACTGAATCT-3' TGTAGTAACCTAACAGGAGGTAT-3'; $H s f 21$, 5'-GCCAGCTTAACACATATGGT-3' and 5'-TCTGATTATTCATTCTCACTCGT-3'; EDS5, 5'-GGTACATTGCTGGCGG-3' 
and

5'-GTATGCCTCCAGGCGA-3';

At3g60420,

5'-AGATCAAGGTGGCTATTGAA-3' and 5'- CTCAAAGGCTTGTGCAG-3'; MYB29,

5'-TTCTCGCGGCAACAAG-3' and 5'- GCTGGTTATCTCCGGTACA-3'; Actin2/8,

5'-GGTAACATTGTGCTCAGTGGTGG-3'

and

5'-AACGACCTTAATCTTCATGCTGC-3';

ICS1,

5'-

ATGAGATtCAGCCTCGCTGT-3' and 5'-TGATGGATCTCCAATCGTCA-3'; $P R-1$, 5'- ATTACTTCATTAGTATGGCTTCT-3' and 5'-CTTGTCTGGCGTCTCC-3'. All kits were used according to the manufacture's protocols.

\section{Microarray analysis}

Ten-day-old seedlings of wild type and atnfxll mutant plants were grown on MS plates

and harvested after mock or $1 \mu \mathrm{M}$ T-2 toxin treatment for $24 \mathrm{hr}$. Samples for microarray analysis were taken at the middle stage of the light period. Total RNA was prepared from T-2 toxin-treated or untreated Arabidopsis shoots using a guanidine hydrochloride-phenol-chloroform extraction method, as previously described (Nishiuchi et al., 2006). The quality of RNA was assessed using the RNA 6000 Nano 
LabChip Kit (Bioanalyzer 2100; Agilent Technologies, Inc.), then the microarray experiment was carried out using the Agilent Arabidopsis 1 Oligo Microarray (Agilent Technologies, Inc.), according to the Agilent 60-mer Oligo Microarray Processing Protocol (Agilent Technologies, Inc.). Total RNA (5 $\mu \mathrm{g})$ from wild type and atnfxl1 mutant plants was used to prepare Cy3- and Cy5-labeled cDNAs, respectively, using a Fluorescent Direct Labeling Kit (Agilent Technologies). The two different fluorescently labeled cDNAs were combined and purified using an RNeasy RNA purification Kit (Qiagen Inc.). Following hybridization and washing, arrays were scanned under maximum laser intensity with both the $\mathrm{Cy} 3$ and $\mathrm{Cy} 5$ channels using an Agilent microarray scanner (G2565BA; Agilent Technologies). Images were analyzed with Feature Extraction Software (version 7.0; Agilent Technologies). Two independent experiments were carried out using different plant samples to demonstrate the reproducibility of the microarray analysis. Upregulated or downregulated genes were designated as such if a 3-fold or greater change in expression relative to wild type plants was observed. All changes in gene expression were statistically significant $(\mathrm{P}<0.01)$. 


\section{SA measurement.}

SA and SAG levels in mock- or T-2 toxin-treated samples were measured as described previously (Nakashita et al., 2002).

\section{GUS assays}

For GUS staining, plants were continuously treated with the indicated substance for 8 days. The AtNFXL1 promoter::GUS transformants were fixed in $90 \%$ acetone at $-20^{\circ} \mathrm{C}$, then incubated in a solution containing $0.5 \quad \mathrm{mM} \quad \mathrm{K}_{4}\left[\mathrm{Fe}(\mathrm{CN})_{6}\right], \quad 0.5 \quad \mathrm{mM}$ $\mathrm{K}_{4}\left[\mathrm{Fe}(\mathrm{CN})_{6}\right] \cdot 3 \mathrm{H}_{2} \mathrm{O}, 1 \mathrm{mM}$ EDTA, and $1 \mathrm{mM} \mathrm{X}$-Gluc in $100 \mathrm{mM}$ phosphate buffer (pH7.2) at $37^{\circ} \mathrm{C}$ for $2 \mathrm{hr}$. Samples were destained by a series of ethanol washes. For the fluorometric assay, 8-day-old plants were transferred to medium containing the indicated substance, incubated for $24 \mathrm{hr}$, and then subjected to quantification of GUS activity. The fluorometric assay of GUS activity was performed as previously described (Nishiuchi et al., 1995).

\section{Bacterial Infection}


The PstDC3000 infection assay was performed as previously described (Yasuda et al., 2003).

\section{Visualization of the GFP-AtNFXL1 fusion protein.}

The entire coding region of AtNFXL1 was amplified from cDNA by PCR using the following primers: 5'-CACCATGAGCTTTCAAGTCAGGCG-3' and 5'-TCACTCACATACCTTCTCCC-3'. The PCR fragment was inserted into the $\mathrm{pENTR}^{\mathrm{TM}} / \mathrm{D}-\mathrm{TOPO}$ entry vector (Invitrogen Inc, Germany), then introduced into pH7WGF2 (Karimi et al., 2002). Protoplasts of Arabidopsis T87 suspension culture cells were transiently transfected with the GFP-AtNFXL1 plasmid using the polyethylene glycol (PEG) method (Abel and Theologis, 1994). GFP was visualized by microscopy (BX-50; Olympus Optical, Tokyo) using a built-in BX-FLA epifluorescent unit.

\section{Acknowledgement}

We thank Dr. Hiroaki Ichikawa for kindly providing the binary vector, pSMAH621

containing the hygromycin-resistance gene $(h p t)$ as a selection marker. 


\section{References}

Abel, S. and Theologis, A. (1994) Transient transformation of Arabidopsis leaf protoplasts: a versatile experimental system to study gene expression. Plant J. 5, $421-427$.

Asai, T., Tena, G., Plotnikova, J., Willmann, M.R., Chiu, W.L., Gomez-Gomez, L., Boller, T., Ausubel, F.M. and Sheen, J. (2002) MAP kinase signalling cascade in Arabidopsis innate immunity. Nature 415, 977-983.

Asano, T., Yoshioka, Y., Kurei, S., Sakamoto, W., Sodmergen and Machida, Y. (2004) A mutation of the CRUMPLED LEAF gene that encodes a protein localized in the outer envelope membrane of plastids affects the pattern of cell division, cell differentiation, and plastid division in Arabidopsis. Plant J. 38, 448-459.

Bai, G.H., Desjardins, A.E. and Plattner, R.D. (2001) Deoxynivalenol-nonproducing Fusarium graminearum causes initial infection, but does not cause disease spread in wheat spikes. Mycopathologia 153, 91-98. 
Baker, S.S., Wilhelm, K.S. and Thomashow, M.F. (1994) The 5'-region of Arabidopsis thaliana cor15a has cis-acting elements that confer cold-, drought- and ABA-regulated gene expression. Plant Mol. Biol. 24, 701-713.

Bowling, S.A., Guo, A., Cao, H., Gordon, A.S., Klessig, D.F. and Dong, X. (1994) A mutation in Arabidopsis that leads to constitutive expression of systemic acquired resistance. Plant Cell 6, 1845-1857.

Chen, C. and Chen, Z. (2002) Potentiation of developmentally regulated plant defense response by AtWRKY18, a pathogen-induced Arabidopsis transcription factor. Plant Physiol. 129, 706-716.

Desjardins, A.E., Bai, G., Plattner1, R.D. and Proctor1, R.H. (2000) Analysis of aberrant virulence of Gibberella zeae following transformation-mediated complementation of a trichothecene-deficient (Tri5) mutant. Microbiology 146, 2059-2068.

Dong, J., Chen, C. and Chen, Z. (2003) Expression profiles of the Arabidopsis WRKY gene superfamily during plant defense response. Plant Mol. Biol. 51, 21-37.

Eudes, F., Comeau, A., Rioux, S. and Collin, J. (2001) Impact of trichothecenes on 
Fusarium head blight [Fusarium graminearum] development in spring wheat (Triticum aestivum). Can. J. Plant Pathol. 23, 318-322.

Eulgem, T., Rushton, P.J., Robatzek, S. and Somssich, I.E. (2000) The WRKY superfamily of plant transcription factors. Trends Plant Sci. 5, 199-206.

Gewin, L., Myers, H., Kiyono, T. and Galloway, A.D. (2004) Identification of a novel telomerase repressor that interacts with the human papillovirus type-16 E6/E6-AP complex. Genes Dev. 18, 2269-2282.

Kimura, M., Takahashi-Ando, N., Nishiuchi, T., Ohsato, S., Tokai, T., Ochiai, N., Fujimura, M., Kudo, T., Hamamoto, H. and Yamaguchi, I. (2006) Molecular biology and biotechnology for reduction of Fusarium mycotoxin contamination. Pestic. Biochem. and Physiol. 86, 117-123.

Karimi, M., Inze, D. and Depicker, A. (2002) Gateway vectors for Agrobacterium-mediated plant transformation. Trends Plant Sci. 7, 193-195.

Kunz, J., Loeschmann, A., Deuter-Reinhard, M. and Hall, M.N. (2000) FAP1, a homologue of human transcription factor NF-X1, competes with rapamycin for binding to FKBP12 in yeast. Mol. Microbiol. 37, 1480-1493. 
Kurkela, S. and Franck, M. (1990) Cloning and characterization of a cold- and ABA-inducible Arabidopsis gene. Plant Mol. Biol. 15, 137-144.

Lang, V. and Palva, E.T. (1992) The expression of a $r a b$-related gene, $r a b 18$, is induced by abscisic acid during the cold acclimation process of Arabidopsis thaliana (L.) Heynh. Plant Mol. Biol. 20, 951-962.

Lisso, J., Altmann, T. and Mussig, C. (2006) The AtNFXL1 gene encodes a NF-X1 type zinc finger protein required for growth under salt stress. FEBS Lett. 580, $4851-4856$.

Liu, Y., Schiff, M. and Dinesh-Kumar, S.P. (2004) Involvement of MEK1 MAPKK, NTF6 MAPK, WRKY/MYB transcription factors, COII and CTR1 in N-mediated resistance to tobacco mosaic virus. Plant J. 38, 800-809.

Lorick, K.L., Jensen, J.P., Fang, S., Ong, A.M., Hatakeyama, S. and Weissman, A.M. (1999) RING fingers mediate ubiquitin-conjugating enzyme (E2)-dependent ubiquitination. Proc. Natl. Acad. Sci. 96, 11364-11369.

Masuda, D., Ishida, M., Yamaguchi, K., Yamaguchi, I., Kimura, M. and Nishiuchi, T. (2007) Phytotoxic effects of trichothecenes on the growth and morphology in 
Arabidopsis thaliana. J. Exp. Bot. 58, 1617-1626.

Mauch-Mani, B. and Mauch, F. (2005) The role of abscisic acid in plant-pathogen interactions. Curr. Opin. Plant Biol. 8, 409-414.

Nawrath, C., Heck, S., Parinthawong, N. and Metraux, J.P. (2002) EDS5, an essential component of salicylic acid-dependent signaling for disease resistance in Arabidopsis, is a member of the MATE transporter family. Plant Cell 14, 275-286.

Nishiuchi, T., Masuda, D., Nakashita, H., Ichimura, K., Shinozaki, K., Yoshida, S., Kimura, M., Yamaguchi, I. and Yamaguchi, K. (2006) Fusarium phytotoxin trichothecenes have an elicitor-like activity in Arabidopsis thaliana, but the activity differed significantly among their molecular species. Mol. Plant Microbe Interact. 19, $512-20$.

Nishiuchi, T., Nakamura, T., Abe, T., Kodama, H., Nishimura, M. and Iba, K. (1995) Tissue-specific and light-responsive regulation of the promoter region of the Arabidopsis thaliana chloroplast $\omega-3$ fatty acid desaturase gene (FAD7). Plant Mol. Biol. 29, 599-609. 
Robatzek, S. and Somssich, I.E. (2002) Targets of AtWRKY6 regulation during plant senescence and pathogen defense. Genes Dev. 16, 1139-1149.

Rogers, E.E., and Ausubel, F.M. (1997) Arabidopsis enhanced disease susceptibility mutants exhibit enhanced susceptibility to several bacterial pathogens and alterations in PR-1 gene expression. Plant Cell 9, 305-316.

Serrano, M. and Guzman, P. (2004) Isolation and gene expression analysis of Arabidopsis thaliana mutants with constitutive expression of $A T L 2$, an early elicitor-response RING-H2 zinc-finger gene. Genetics 167, 919-29.

Shifrin, V.I. and Anderson, P. (1999) Trichothecene mycotoxins trigger a ribotoxic stress response that activates c-Jun N-terminal kinase and p38 mitogen-activated protein kinase and induces apoptosis. J. Biol. Chem. 274, 13985-13992.

Song, Z., Krishna, S., Thanos, D., Strominger, J.L. and Ono, S.J. (1994) A novel cysteine-rich sequence-specific DNA-binding protein interacts with the conserved X-box motif of the human major histocompatibility complex class II genes via a repeated Cys-His domain and functions as a transcriptional repressor. J. Exp. Med. 180, $1763-1774$. 
Stroumbakis, N.D., Li, Z. and Tolias, P.P. (1996) A homolog of human transcription factor NF-X1 encoded by the Drosophila shuttle craft gene is required in the embryonic central nervous system. Mol. Cell Biol. 16, 192-201.

Sudakin, D.L. (2003) Trichothecenes in the environment: relevance to human health. Toxicol. Lett. 143. 97-107.

Takai, R., Matsuda, N., Nakano, A., Hasegawa, K., Akimoto, C., Shibuya, N. and Minami, E. (2002) EL5, a rice $N$-acetylchitooligosaccharide elicitor-responsive RING-H2 finger protein, is a ubiquitin ligase which functions in vitro in co-operation with an elicitor-responsive ubiquitin-conjugating enzyme, OsUBC5b. Plant J. 30, 447-455.

Ulker, B. and Somssich, I.E. (2004) WRKY transcription factors: from DNA binding towards biological function. Curr. Opin. Plant Biol. 7, 491-498.

Urban, M., Daniels, S., Mott, E. and Hammond-Kosack, K. (2002) Arabidopsis is susceptible to the cereal ear blight fungal pathogens Fusarium graminearum and Fusarium culmorum. Plant J. 32, 961-973. 
Ward, T.J., Bielawski, J.P., Kistler, H.C., Sullivan, E. and O'Donnell, K. (2002)

Ancestral polymorphism and adaptive evolution in the trichothecene mycotoxin gene

cluster of phytopathogenic Fusarium. Proc. Natl. Acad. Sci. 99, 9278-9283.

Wildermuth, M.C., Dewdney, J., Wu, G. and Ausubel, F.M. (2001) Isochorismate synthase is required to synthesize salicylic acid for plant defence. Nature 414, 562-565.

Yasuda, M., Nakashita, H., Hasegawa, S., Nishioka, M., Arai, Y., Uramoto, M., Yamaguchi, I. and Yoshida, S. (2003) N-cyanomethyl-2-chloroisonicotinamide induces systemic acquired resistance in Arabidopsis without salicylic acid accumulation. Biosci. Biotechnol. Biochem. 67, 322-328. 


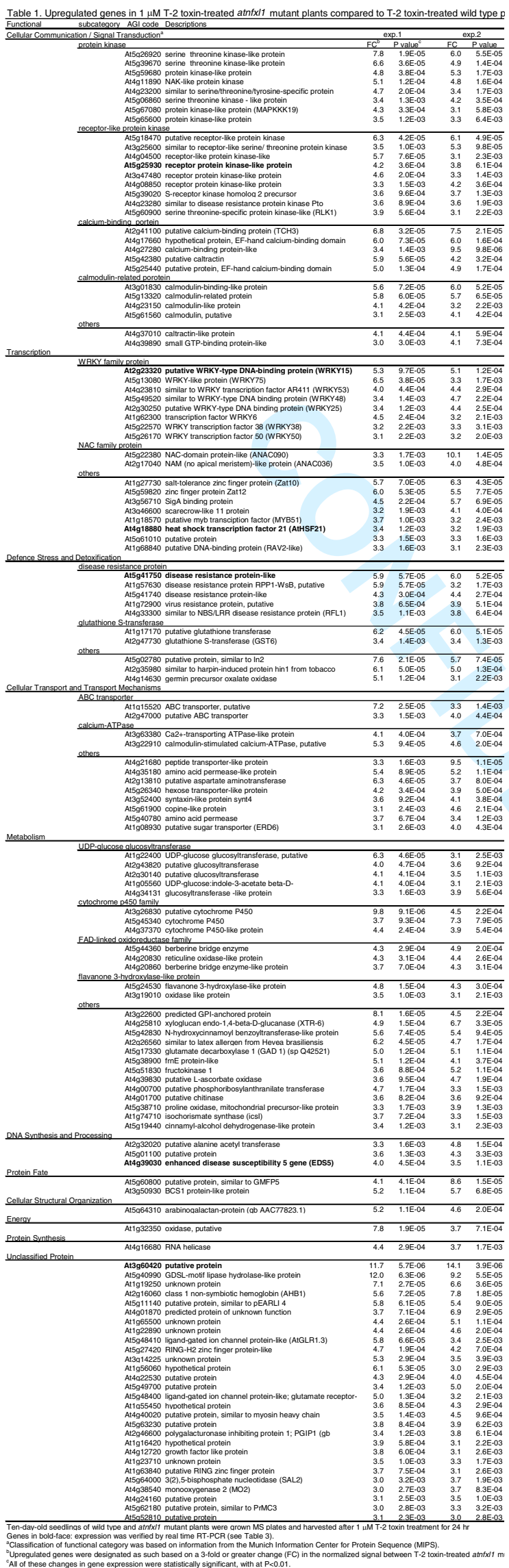


Table 2. Downregulated genes in the T-2 toxin-treated atnfx/1 mutant plants compared to T-2 toxin-treated w

\begin{tabular}{|c|c|c|c|c|c|}
\hline & & \multicolumn{2}{|c|}{ exp.1 } & \multicolumn{2}{|c|}{ exp.2 } \\
\hline AGI code & Descriptions & $\mathrm{FC}^{\mathrm{a}}$ & $\mathrm{P}$ value $^{\mathrm{b}}$ & $\mathrm{FC}$ & P value \\
\hline$\overline{\text { At4g19170 }}$ & neoxanthin cleavage enzyme-like protein & 0.164 & $5.6 \mathrm{E}-03$ & 0.073 & $2.0 \mathrm{E}-03$ \\
\hline At4g16830 & nuclear antigen homolog & 0.111 & 3.3E-03 & 0.164 & $5.7 \mathrm{E}-03$ \\
\hline At5g50950 & fumarate hydratase & 0.150 & $4.8 \mathrm{E}-03$ & 0.137 & 4.3E-03 \\
\hline At5g23010 & 2-isopropylmalate synthase-like & 0.206 & 8.7E-03 & 0.090 & $2.5 \mathrm{E}-03$ \\
\hline At4g13770 & cytochrome P450 monooxygenase (CYP83A1) & 0.193 & 7.7E-03 & 0.111 & $3.2 \mathrm{E}-03$ \\
\hline At5g07690 & myb family transcription factor (MYB29) & 0.200 & 8.7E-03 & 0.116 & 3.6E-03 \\
\hline At1g14250 & nucleoside phosphatase family protein / GDA1/CD39 family protein & 0.200 & 8.3E-03 & 0.128 & 4.0E-03 \\
\hline At5g03760 & glycosyl transferase family 2 protein & 0.161 & $5.8 \mathrm{E}-03$ & 0.174 & 7.3E-03 \\
\hline At4g21650 & subtilisin proteinase - like & 0.206 & 8.9E-03 & 0.151 & $5.0 \mathrm{E}-03$ \\
\hline At3g27690 & chlorophyll A-B binding protein (LHCB2:4) & 0.196 & $7.9 \mathrm{E}-03$ & 0.164 & $5.6 \mathrm{E}-03$ \\
\hline At5g12250 & tubulin beta- 6 chain & 0.189 & 7.3E-03 & 0.186 & 7.2E-03 \\
\hline At4g21960 & peroxidase 42 (PER42) & 0.217 & $9.8 \mathrm{E}-03$ & 0.199 & $8.1 \mathrm{E}-03$ \\
\hline
\end{tabular}
Ten-day-old seedlings of wild type and atnfxl1 mutant plants were grown MS plates and harvested after mock or $1 \mu \mathrm{M}$ T-2 toxin treatment The expression of MYB29 (bold-face) was verified by real time RT-PCR analysis (see Table 3).

${ }^{a}$ Downregulated genes were designated as such based on a 3-fold or greater change in the normalized signal of T-2 toxin-treated atnfx/1 n ${ }^{\mathrm{b}} \mathrm{All}$ of these changes in gene expression were statistically significant, with at $\mathrm{P}<0.01$. 
Table 3. Validation of microarray results in the $1 \mu \mathrm{M} \mathrm{T}-2$ toxin-treated plants by real time PCR.

\begin{tabular}{|c|c|c|c|}
\hline \multirow[b]{2}{*}{ AGI code } & \multicolumn{2}{|c|}{ old change (atnfx/1 mutant vs wild type) } & \multirow[b]{2}{*}{ Description } \\
\hline & Microarray $^{\mathrm{a}}$ & real time $\mathrm{PCR}^{\mathrm{b}}$ & \\
\hline \multicolumn{4}{|l|}{ Upregulated Genes } \\
\hline At5g25930 & 4.00 & $11.6 \pm 1.39$ & receptor protein kinase-like protein \\
\hline At2g23320 & 5.17 & $4.87 \pm 0.26$ & putative WRKY-type DNA-binding protein (WRKY15) \\
\hline At4g18880 & 3.30 & $5.21 \pm 0.68$ & heat shock transcription factor 21 (AtHSF21) \\
\hline At5g41750 & 5.97 & $12.08 \pm 1.56$ & disease resistance protein-like \\
\hline At4g39030 & 3.76 & $7.52 \pm 1.04$ & enhanced disease susceptibility 5 gene (EDS5) \\
\hline At3g60420 & 12.84 & $11.56 \pm 2.16$ & putative protein \\
\hline \multicolumn{4}{|c|}{ Downregulated genes } \\
\hline At5g07690 & 0.15 & $0.12 \pm 0.02$ & Myb family transcription factor (MYB29) \\
\hline
\end{tabular}




\section{Figure legends}

Figure 1. An atnfxl1 mutant (atnfxl1-1) is hypersensitive to type A trichothecenes. (a)

Schematic diagram of AtNFXL1 in Arabidopsis thaliana. Boxes indicate exons. The organization of the exon-intron boundary was predicted by the nucleotide sequence of the full length cDNA, and is identical to our results. The T-DNA insertion site is indicated by a triangle. Two different regions (basepairs 120-438 and 2368-3568) of AtNFXL1 for RT-PCR analysis is indicated by thick lines. (b) A truncated transcript of AtNFXL1 was observed in the atnfxll-1 mutant. Ten-day-old seedlings of wild type and atnfxl1-1 mutant plants were grown on MS plates and harvested after mock treatment, or $1 \mu \mathrm{M}$ T-2 toxin treatment for $24 \mathrm{hr}$. Total RNA was prepared from the seedlings and used for RT-PCR analysis. Two different regions (basepairs 120-438 and 2368-3568) of AtNFXL1 were amplified by specific primer sets. Actin2/8 was used as a loading control. (c) Representative photographs of wild type, atnfxl1, and complementation plant lines that were mock-treated (upper row), or treated with $0.1 \mu \mathrm{M}$ T-2 toxin (lower row). Sterile seeds were sown on MS medium with or without $0.1 \mu \mathrm{M} \mathrm{T}-2$ toxin, and then stratified for $2 \mathrm{~d}$ at $4^{\circ} \mathrm{C}$ in the dark. Plants were grown for 8 days in a growth chamber, 
and then photographed. Scale bars $=1 \mathrm{~cm}$. (d) The fresh weight of each plant is expressed relative (\%) to mock-treated wild type. Plants were treated with $0.1 \mu \mathrm{M}$ T-2 toxin or $10 \mu \mathrm{M}$ DON without trichothecenes, as stated above. atnfxl1:PAtNFXL1::AtNFXL1 (line \#5) refers to an atnfxl1 mutant carrying an AtNFXL1 promoter::AtNFXL1 gene fusion. Data is representative of two independent experiments. *, $P<0.01$, based on the Student's $t$-test. Similar results were obtained in other six independent complementation lines.

Figure 2. AtNFXL1 is involved in SA biosynthesis and expression of SA-related genes. Eight-day-old plants were either mock-treated or treated with $1 \mu \mathrm{M} \mathrm{T}-2$ toxin for $24 \mathrm{hr}$ and then subjected to Real time PCR analysis (a) or SA quantification (b-c). (a) Real time PCR analysis of PR-1 and ICS1 of atnfxl1 mutant and wild type plants. Total RNA was isolated from each sample and then subjected to Real time PCR analysis. The levels of mRNA were determined by real-time RT-PCR, and normalized with that of Actin2/8. Expression levels are relative to that of mock-treated wild type samples. Data is the average of three independent samples. Error bars indicate the standard deviation. (b-c) 
Enhanced accumulation of SA in T-2 toxin-treated atnfxll mutant plants. (b) Free and (c) total SA levels were quantified by high-performance liquid chromatography (data represents the means \pm standard deviation, $n=4$ ).

Figure 3. GUS staining and quantification of GUS activity in AtNFXL1 promoter::GUS stable transformants in response to elicitor, phytohormone, or trichothecene treatment. GUS staining of mock- (a), T-2 toxin- (b) or SA-treated 8 day old plants (c). Sterile seeds were sown on MS agar medium with $0.1 \mu \mathrm{M}$ T-2 or $100 \mu \mathrm{M}$ SA, and then stratified for $2 \mathrm{~d}$ at $4^{\circ} \mathrm{C}$ in the dark. Plants were grown for 8 days in a growth chamber, and then subjected to GUS staining. Scale bars $=1 \mathrm{~mm}$. (d) Quantification of GUS activity in AtNFXL1 promoter::GUS stable transformants treated with the indicated substances. Plants were grown for 8 days on MS agar medium in a growth chamber, and then either mock-treated or treated with the indicated substance for $24 \mathrm{hr}$ and used for quantitative GUS assays. GUS activity in treated samples relative to mock-treated samples was measured using a fluorometric GUS assays $(n=4)$. Data is representative of two independent experiments. 
Figure 4. Reduced susceptibility of atnfxll mutant plants to the compatible pathogen Pst DC3000. (a) Leaves of atnfxl1 mutant (closed circles) and wild type (open circles) plants were collected $0,1,2$ and 4 days post-inoculation and homogenized in $10 \mathrm{mM}$ $\mathrm{MgCl}_{2}$. The number of colony-forming units (CFU) was estimated by growth on nutrient broth agar plates after the appropriate dilution. Data represents the averages \pm standard deviation $(\mathrm{n}=6)$. A significant difference between wild type and atnfxl1 mutant plants was observed in the number of CFU/g fresh weight ( $\mathrm{p}<0.05$, ANOVA). Data is representative of two independent experiments. (b) Complementation analysis of the reduced susceptibility to $P$ stDC3000 in an atnfxll mutant. Leaves of wild type, atnfxl1, and complementation plant line were collected 2 days post-inoculation. Data represents the averages \pm standard deviation $(n=6)$. A significant difference between the number of CFU/g fresh weight of atnfxll mutant plants and wild type/complementation line \#4 plants was observed $(\mathrm{p}<0.05$, ANOVA $)$. Similar results were obtained in complementation lines \#1 and \#3. 
Figure 5. Model of opposing functions of AtNFXL1 in biotic and abiotic stress response.

Biotic stress often causes accumulation of elicitors and/or SA in host plants. SA and elicitors including T-2 toxin induce the expression of AtNFXL1. An atnfxl1 mutant exhibits a hypersensitivity phenotype to T-2 toxin due to excessive defense responses. AtNFXL1 functions as a negative regulator of defense-related genes via an SA-dependent signaling pathway, which resulting in reduced susceptibility to a virulent pathogen, Pst DC3000 in the atnfxl1 mutant. Abiotic stress such as salt and osmotic stress also induces the expression of AtNFXL1 (Lisso et al., 2006). AtNFXL1 functions as a positive regulator of salt-responsive genes (Lisso et al., 2006). The atnfxl1 mutant exhibited a reduced survival rate under salt stress (Lisso et al., 2006).

Supplemental Figure 1. AtNFXL1 belongs to the NF-X1 family of proteins.

(a) Schematic diagram of AtNFXL1 in Arabidopsis thaliana (Accession no. AAD32867) and comparison of AtNFXL-1 with the following homologues: OsNF-X1, Oryza sativa (Accession no. BAD46154); NF-X1, Homo sapiens (Accession no. NP_002495); STC, Drosophila melanogaster (Accession no. NP_476599); Fap1, 
Saccharomyces cerevisiae (Accession no. NP_014375). The purple regions indicate the

NLS; red and blue indicate the RING-CH finger domain and the nine NF-X1-type Zn finger domains, respectively; green indicates the $\mathrm{R} 3 \mathrm{H}$ domain. (b) A rooted maximum-likelihood phylogenetic tree of AtNFXL1 and AtNFXL1 homologues. DrNF-X1, Danio rerio (Accession no. XP_690559); MmNF-X1, Mus musculus (Accession no. AAF34700); CeNF-X1, Caenorhabditis elegans (Accession no. NP_498394); and SpNF-X1, Schizosaccharomyces pombe (Accession no. CAA21417). (c) Alignment of the amino acid sequences of the nine AtNFXL1-type $\mathrm{Zn}$ finger domains. The number to the left of each repeat indicates its position in the AtNFXL1 protein sequence. The consensus sequence for the $\mathrm{Zn}$ finger repeat is shown above the sequences, and is based on matches in seven of the nine aligned sequences.

Supplemental Figure 2. Subcellular localization of GFP-AtNFXL1 and GFP proteins in Arabidopsis cells. Protoplasts of Arabidopsis T87 suspension culture cells were transfected using the polyethylene glycol (PEG) method. GFP-AtNFXL1 fusion protein localized to the nucleus of Arabidopsis cells (a-c). In contrast, GFP localized to the 
cytosol (d-f). GFP fluorescence was visualized in using a fluorescence microscope. 
(a)

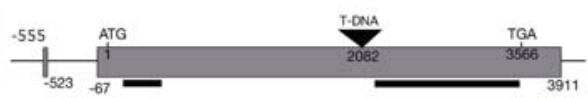

(b)

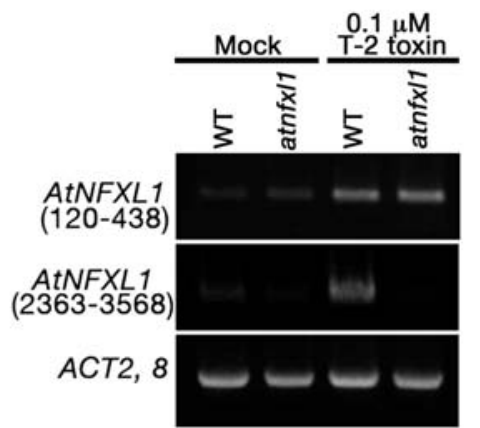

(c)

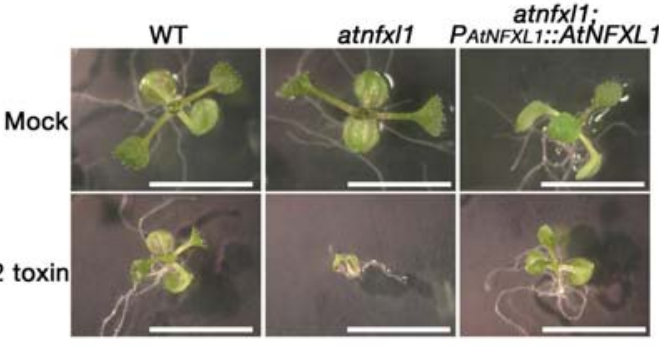

(d)

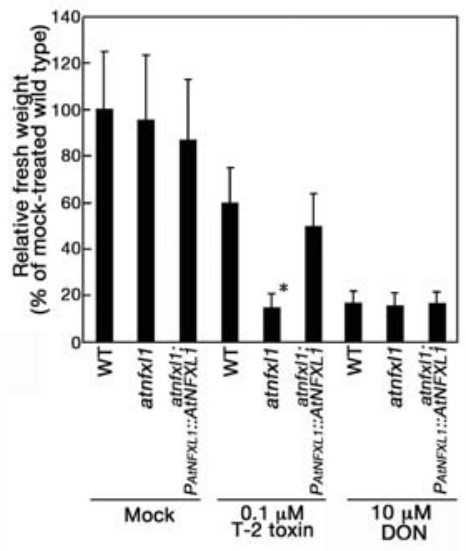

Figure 1

$111 \times 213 \mathrm{~mm}(400 \times 400 \mathrm{DPI})$ 
(a)

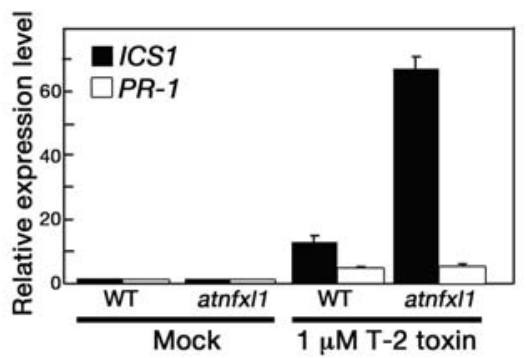

(b)

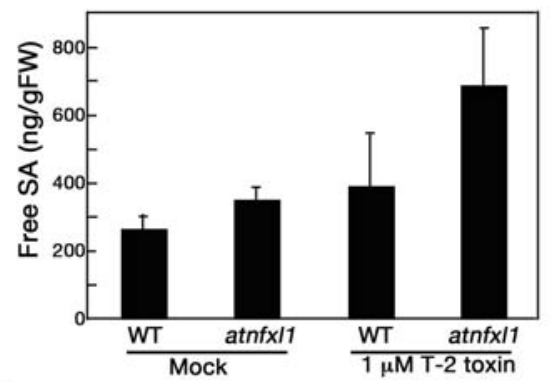

(c)

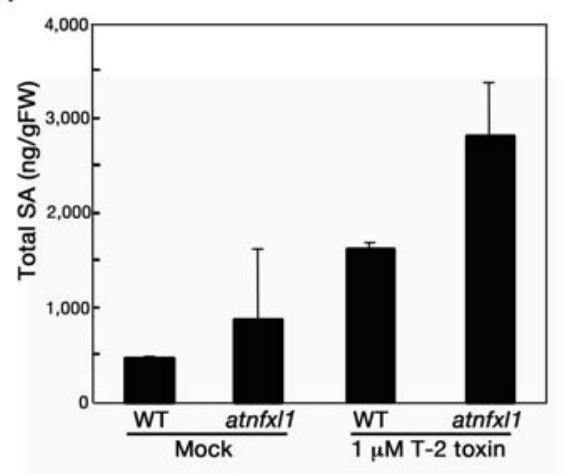

Figure 2

$100 \times 210 \mathrm{~mm}(400 \times 400 \mathrm{DPI})$ 

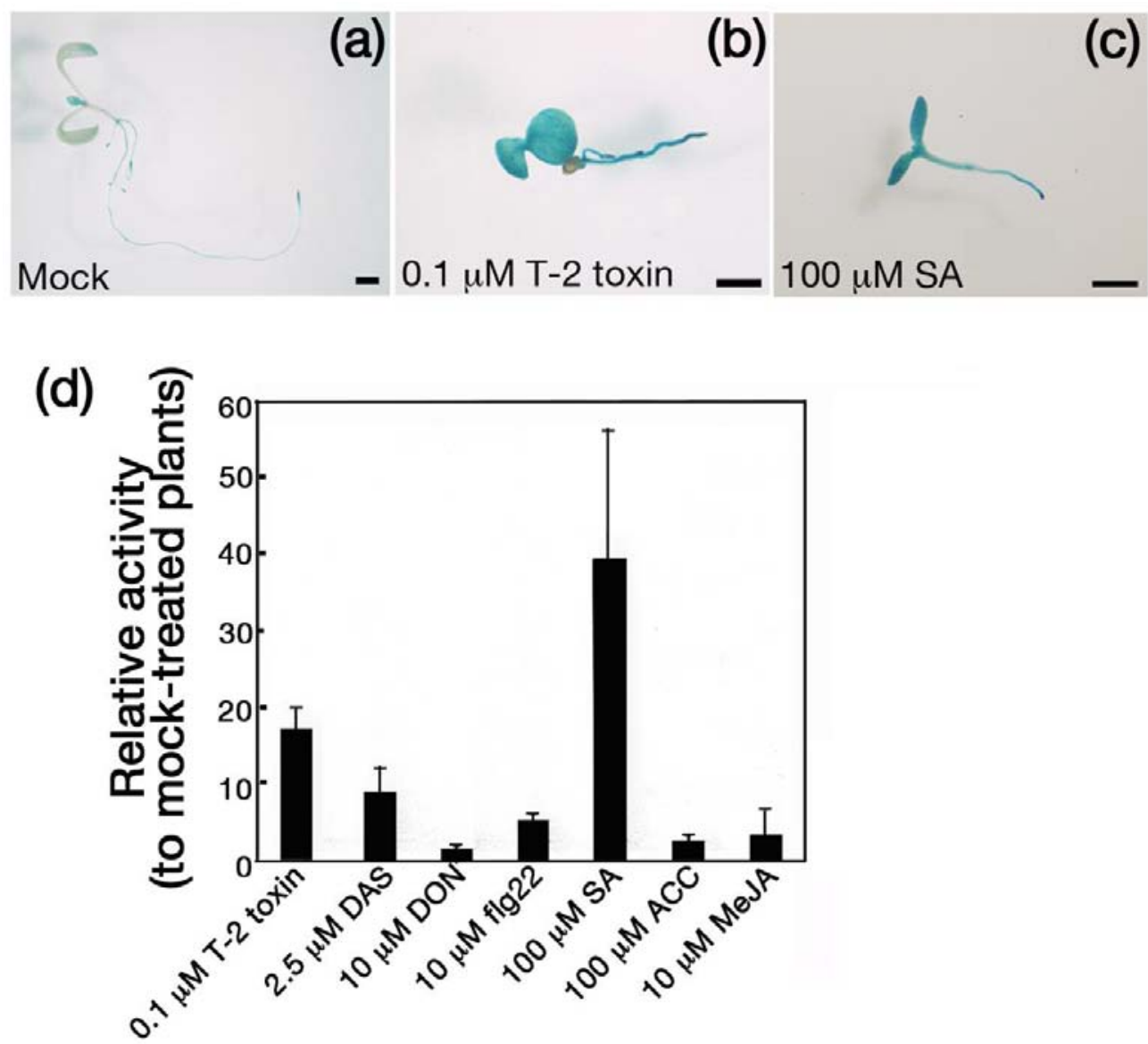

Figure 3

$80 \times 82 \mathrm{~mm}(400 \times 400 \mathrm{DPI})$ 


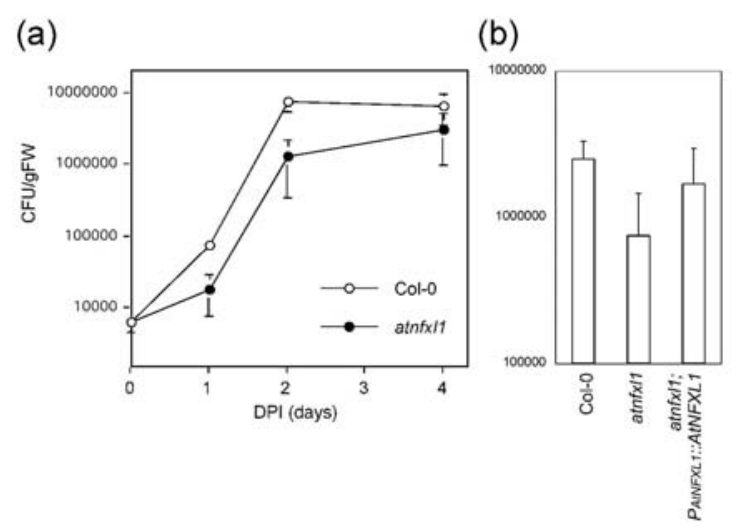

Figure 4 


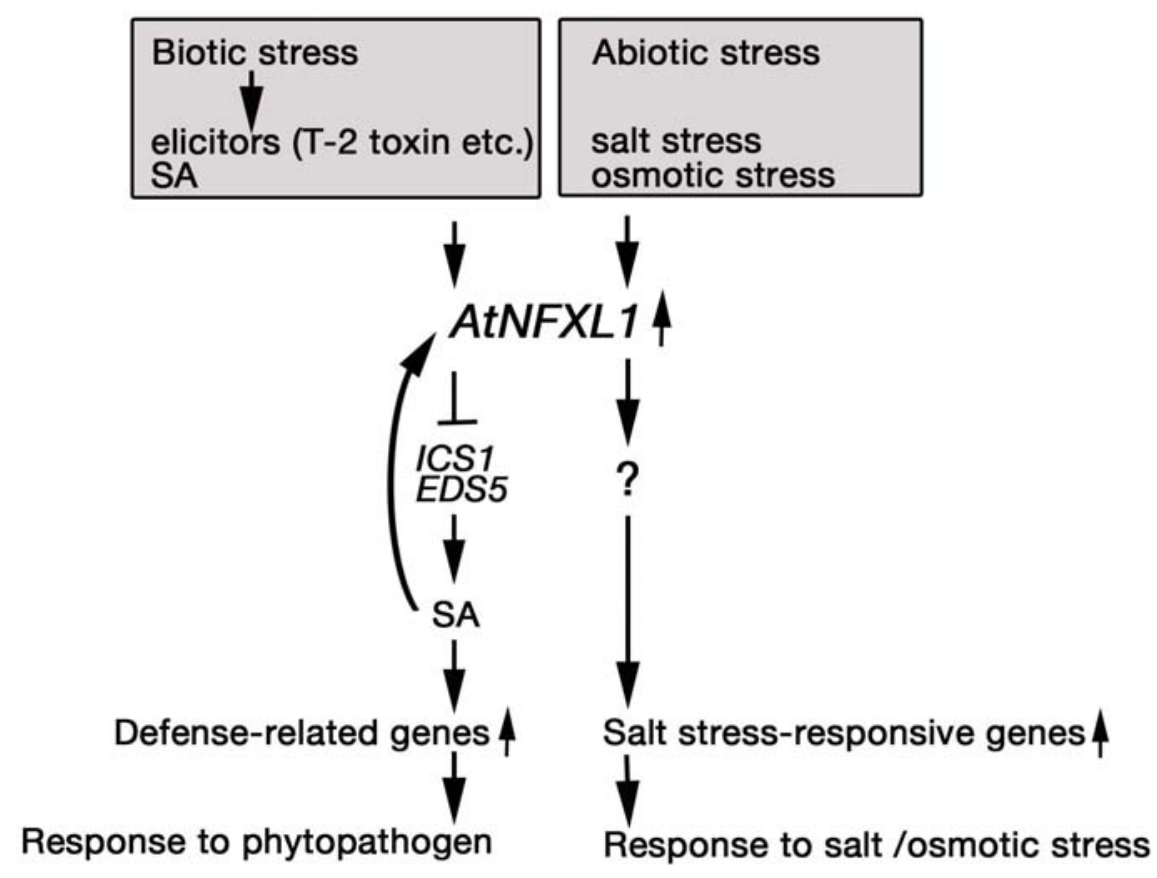

Figure 5

$120 \times 109 \mathrm{~mm}(400 \times 400 \mathrm{DPI})$ 